\title{
Compatibility of pellet fuelling with ELM suppression by RMPs in the ASDEX Upgrade tokamak
}

\author{
M Valovič ${ }^{1}$, P T Lang ${ }^{2}$, A Kirk ${ }^{1}$, W Suttrop ${ }^{2}$, A Bock ${ }^{2}$, P J Mc Carthy ${ }^{3}$, M Faitsch ${ }^{2}$, \\ B Plöck| ${ }^{2}$, the ASDEX Upgrade team ${ }^{2}$ and the EUROfusion MST1 team ${ }^{4}$ \\ ${ }^{1}$ CCFE, Culham Science Centre, Abingdon, OX14 3DB, UK \\ ${ }^{2}$ Max-Planck-Institut für Plasmaphysik, Boltzmannstrasse 2, D-85748 Garching, Germany \\ ${ }^{3}$ Department of Physics, University College Cork, Cork, Ireland \\ E-mail: martin.valovic@ukaea.uk
}

Received xxxxxx

Accepted for publication $\mathrm{xxxxxx}$

Published xxxxxx

\begin{abstract}
It is demonstrated that tokamak plasma can be fuelled by pellets while simultaneously maintaining ELM suppression by external resonant magnetic perturbations (RMPs). Pellets are injected verically from high field site and deposited at outer part of plasma cross section. Each pellet triggers a benign MHD event followed by a short lived ELM-free phase. The ELM suppression phase with pellet fuelling lasts 11 pellet cycles and is terminated by intentionally increasing the pellet rate to cause a transition to the ELMy phase.
\end{abstract}

Keywords: tokamak, pellet fuelling, ELM control

\section{Introduction}

In tokamak fusion reactors such as ITER gas fuelling becomes inefficient and plasma density will be controlled by injection of hydrogen ice pellets [1,2]. Simultaneously Edge Localised Modes (ELMs) have to be avoided to protect exhaust system from large power excursions. One of the ELM control techniques is the application of external Resonant Magnetic Perturbations (RMPs) [3, 4 , 5 , 6 ] and such a system is planned on ITER [7]. Both pellet fuelling and RMPs act on the plasma periphery and therefore it is not surprising that these actuators are coupled as seen on DIII-D [3, 8, 9, 10 ], ASDEX Upgrade $[11,12,13,14]$ and MAST $[15,16]$. This coupling takes the form of two effects. Firstly, application of RMPs increases the peripheral particle transport (density pump out) which in turn has to be compensated by increased pellet fuelling. Secondly, fuelling pellets typically trigger ELMs and thus counteract to ELM control. This letter describes an experiment where plasma is fuelled by pellets and simultaneously full ELM suppression is maintained, for the first time to our knowledge

\section{Experimental setup}

The experiment was performed on ASDEX Upgrade. In order to access the fully stationary ELM suppression phase the upper triangularity has to be elevated to about $\delta_{u}=0.24$

$[17,18,19]$. In addition the plasma has a single null divertor, with radius of the geometric axis $R_{\text {geo }}=1.58 \mathrm{~m}$, horizontal minor radius $a=0.51 \mathrm{~m}$, plasma current $I_{p}=0.94 \mathrm{MA}$, toroidal field $B_{T}(R=1.65 \mathrm{~m})=1.78 T$, safety factor $q_{95}=$ 3.7. The plasma is heated by neutral beams with $P_{N B I}=$ 5.9 MW. ELMs are controlled by RMP coils with toroidal periodicity $n=2$ and for further details see $[17,19])$. 


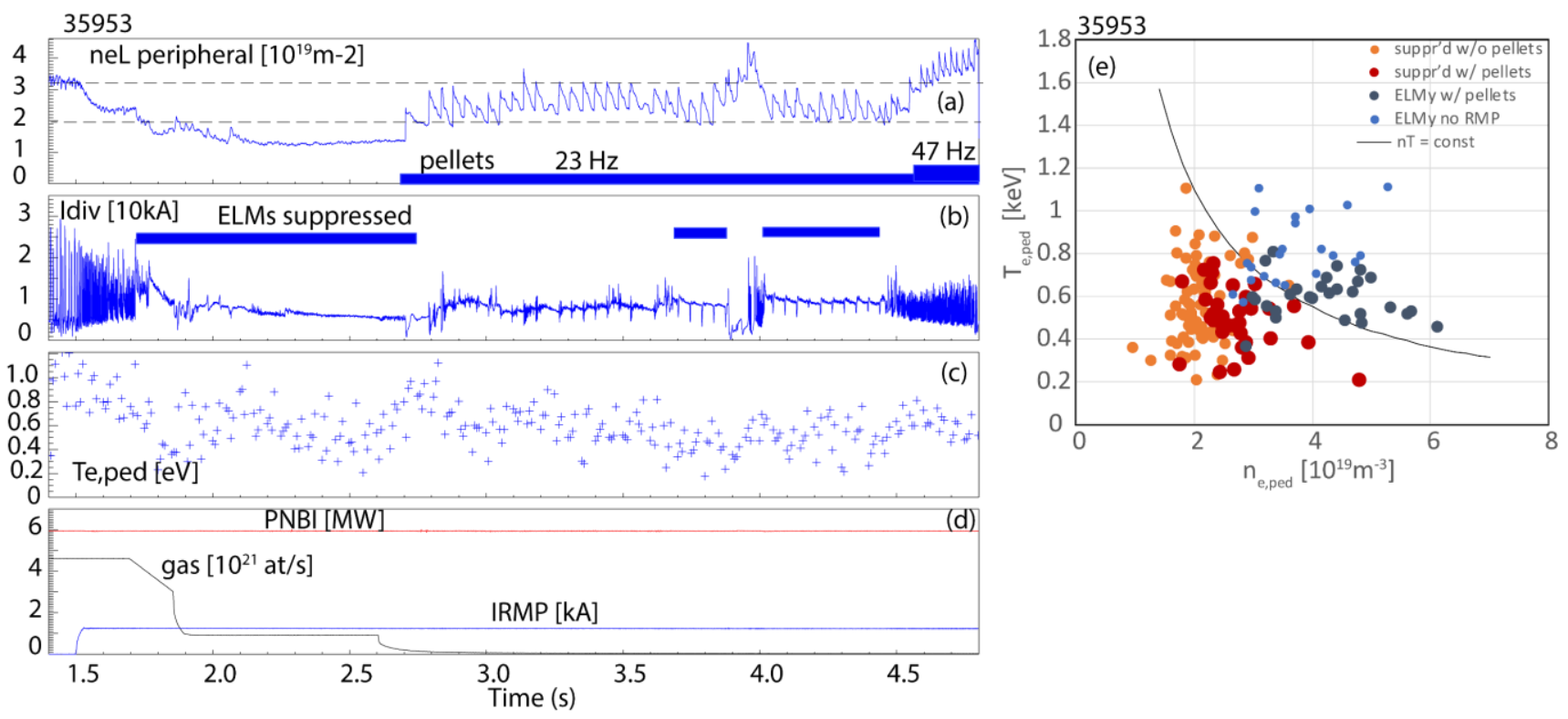

Figure 1. Pellet fuelling of plasma with ELM suppression by RMP. (a) peripheral interferometer signal, (b) divertor tile current - ELMs indicator, (c) electron temperature at pedestal top $\left(\rho_{p o l}=\sqrt{\psi_{N}}=0.91\right)$ by Thomson scattering, (d) NBI power, gas puff rate and RMP current, (e) pedestal top electron temperature and density for different phases of the plasma on the left panels. Each data point represents one Thomson scattering measurement. The borderline phase $2.7 \mathrm{~s}-4.0 \mathrm{~s}$ is omitted for clarity in panel (e).

Pellet fuelling is provided by deuterium pellets injected vertically from the high field side with a velocity of $560 \mathrm{~m} / \mathrm{s}$ and a nominal size of $1.4 \times 1.4 \times 1.5 \mathrm{~mm}$. For this parameter set a total $30 \%$ of the pellet atoms are lost before the pellet arrives in the plasma reducing the effective pellet particle content to $N_{\text {pel }}=1.2 \times 10^{20}$ atoms [19].

Key elements of this experiment are fresh boronisation and gradual reduction of gas fuelling. After application of RMPs the gas fuelling level is reduced to $\Phi_{\text {gas }}=1 \times 10^{21}$ atoms $/ \mathrm{s}$ which allows the ELM suppressed phase to become established (see figure 1). A second gas reduction is introduced just before the pellet train when the gas is completely switched off. The pellet frequency is initially 23 $\mathrm{Hz}$, and later increased to $47 \mathrm{~Hz}$.

\section{Pellet fuelling with suppressed ELMs}

Figure 1 shows the effect of pellet fuelling on a plasma with simultaneous ELM suppression by RMPs. It is seen that during the $23 \mathrm{~Hz}$ pellet phase the peripheral plasma density transiently reaches the pre RMP value (figure 1a). In other words pellet fuelling broadly compensates for the density pump out and switching off the gas fuelling. At present there is no consensus which dimensionless parameter should be matched in order to demonstrate the relevance of this ELM/fuelling control method under ITER conditions. In this situation the selection of the pellet fuelling level that compensates for the density pump out and gas switch off seems to be a reasonable first choice.

Figure 1b shows the divertor tile current that is used as an indicator of ELMs, which manifest themselves as positive spikes. It is seen that during the first part of the pellet train from $2.8 \mathrm{~s}$ to $3.7 \mathrm{~s}$ there are infrequent irregular ELMs (see the positive spikes on the divertor current) indicating partial ELM suppression. This situation spontaneously changes during the second part of the pellet train from $4.1 \mathrm{~s}$ to $4.45 \mathrm{~s}$ where ELMs are completely suppressed. During this phase the density is slightly lower compared to the phase of partial ELM suppression. This is consistent with a notion of an empirical density threshold below which ELM suppression is observed (see the lower horizontal line in figure 1a). This is also in line with the plasma response to the increase of the pellet rate to $47 \mathrm{~Hz}$ which leads to a density increase and transition to ELMy H-mode. Figure 1e shows a reasonable separation between suppressed and ELMy data by the line of constant pressure. Figure 1e also shows that on average the phase with ELM suppression and pellets have slightly (9\%) higher pedestal top pressure compared to ELM suppression without pellets. This is due to increased density at constant temperature, figure $1 \mathrm{a}$, c. This behavior is similar to previous observations with ELM mitigation [14]. Finally note that in figure 2e there are about five ELMy points with pellets which are below the line of constant pressure. These are the data taken immediately after 

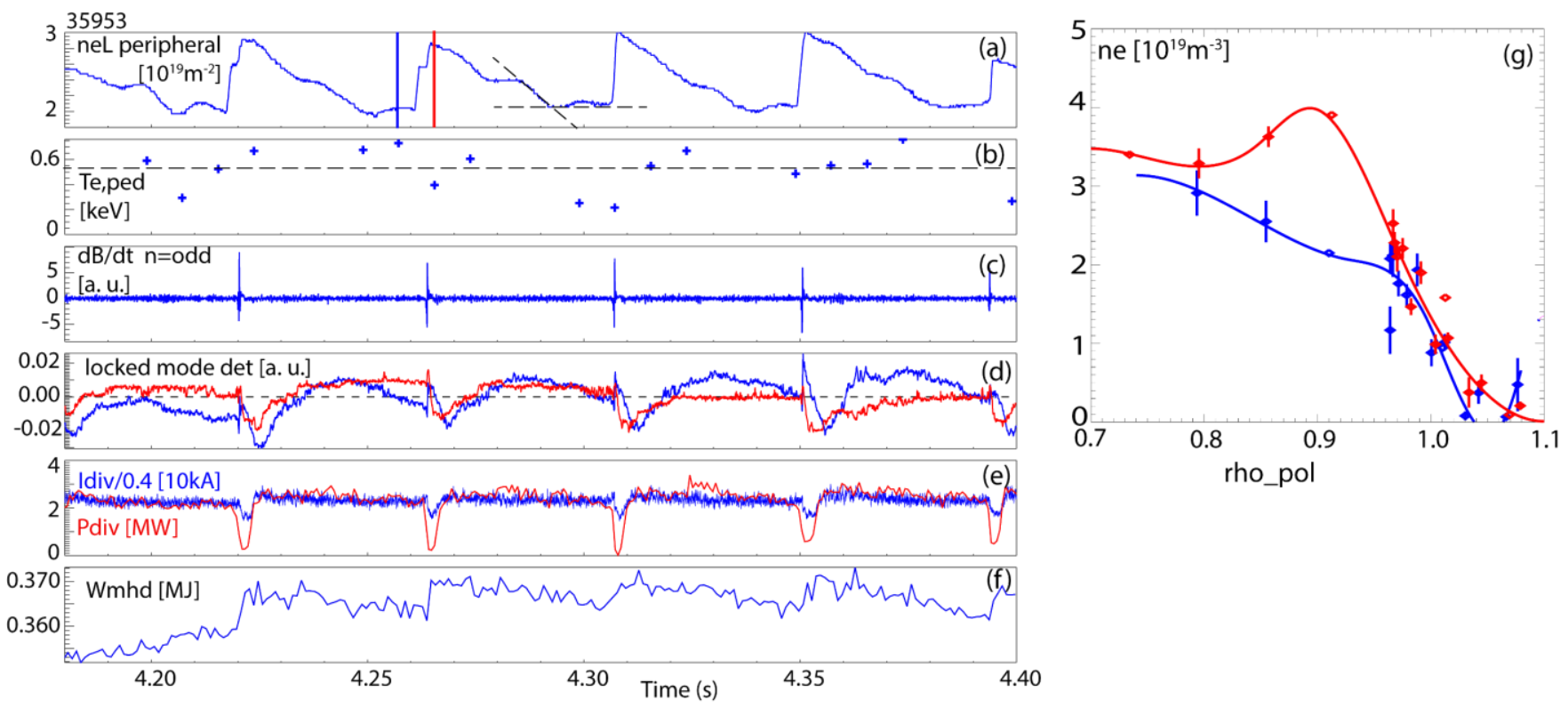

Figure 2. Quasi-stationary ELM suppressed phase (a) peripheral line integral density from the interferometer, (b) pedestal top electron temperature from Thomson scattering at $\rho_{p o l}=\sqrt{\psi_{N}}=0.91$, where $\psi_{N}$ is the normalised poloidal magnetic flux. (c) Mirnov coil signal, (d) locked mode detectors at different toroidal locations, (e) power to the divertor by the infrared camera (red) and divertor tile current (blue), (f) plasma energy content, (g) electron density profiles at times indicated by the vertical lines in panel (a).

the pellet rate is increased to $47 \mathrm{~Hz}$ and as such represent the initial transient phase. All later ELMy data with pellets are above the line of constant pressure.

Figure 2 shows the details of plasma parameters during the quasi-stationary phase with full ELM suppression. The figure 2a shows the peripheral interferometer signal with each pellet causing a sharp density rise. Note that the pellet deposition is peripheral as seen from the electron density profile before and after the pellet in figure $2 \mathrm{~g}$. The maximum of the density perturbation is located at $\rho_{\text {pol }} \sim 0.9$. This is similar to that expected in ITER $[1,21]$, however the ratio of pellet to plasma particles is about a factor of two larger than expected in ITER for fuelling pellets. Regarding the location of $q=m / n$ resonance, the surface $\mathrm{m} / \mathrm{n}=7 / 2$ is localised at $\rho_{\text {pol }}=0.96$ [19], i. e. at the outer part of the pellet deposition.

The trace in figure $2 \mathrm{c}$ shows a magnetic pick up coils signal. It shows that each pellet triggers a short MHD spike. These events are not conventional ELMs. Firstly each such event causes a step like increase of the total plasma stored energy (figure 2f). These short transients are also visible on traces of the power to the divertor measured by the infrared camera showing the short dip after the pellet (see the divertor infrared camera signal in figure $2 \mathrm{f}$ red linne). This behaviour is mirrored by the divertor tile current. All these observations are opposite to conventional ELMs which cause sudden energy loss and a corresponding spikes on a power to the divertor andon the divertor tile current.

To elaborate further on the character of MHD events, figure 3 compares two pellets, one in the phase of ELM suppression and one when ELM suppression is lost due to the increased pellet rate to $47 \mathrm{~Hz}$ (figure 1). It is seen that in both cases the MHD perturbations are synchronous with the pellet ablation light and similar in amplitude. The most striking difference is the divertor tile current $I_{\text {div }}$ which is a measure of power loss to the divertor. In the ELM suppressed case there is a very small increase of the divertor current by $\Delta I_{d i v} \sim 1.5 \mathrm{kA}$ during the MHD perturbation before it drops at the end of the event. In the ELM case $I_{\text {div }}$ significantly increases during the MHD event by $\Delta I_{d i v} \sim 10 \mathrm{kA}$, even displaying a correlation with oscillations on the magnetic signal.This trend is the same on both inner and outer divertor legs. It is not clear whether these two type of events share the same MHD physics with the only difference that in the ELM suppressed case the MHD mode saturates (incomplete ELM). These events are benign and should be compatible with divertor operation.

The transient phases of reduced flux after pellets could be linked to a post pellet density profiles (figure $2 \mathrm{~g}$ ). It is seen that pellets create steep negative density gradient in the zone of $\rho_{\text {pol }}>0.9$, similar to pellet triggered H-mode observed by several tokamaks $[22,23,24]$. It has to be noted that in the 


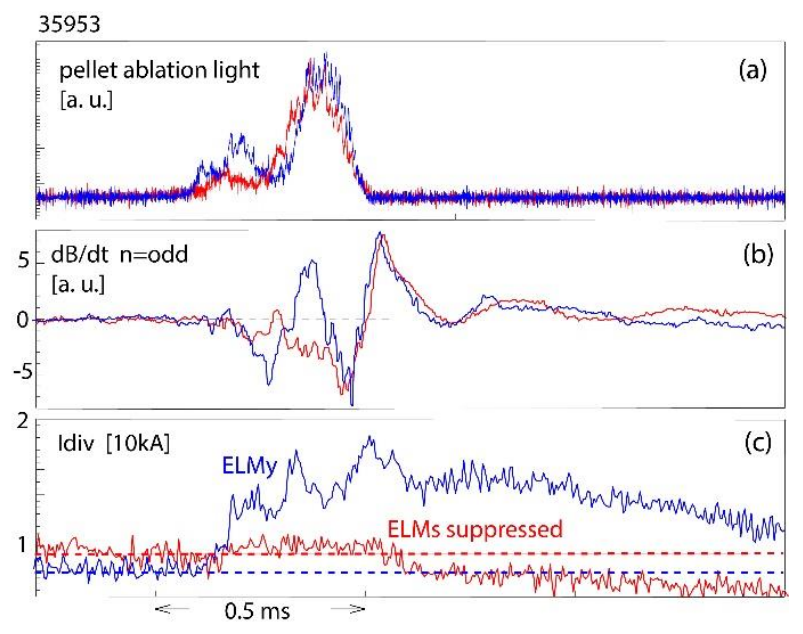

Figure 3. Comparison of pellet triggered magnetic perturbations in the ELMs suppressed phase (red, at $\mathrm{t}_{\mathrm{pel}}=4.2630 \mathrm{~s}$ ) and the ELMy phase (blue, at $\mathrm{t}_{\mathrm{pel}}=4.4795 \mathrm{~s}$ ). (a) pellet ablation light, (b) Mirnov coil signals and (c) divertor current signals. Note that figure shows only initial part of the post pellet reduction of the divertor current.

context of ELM control, these phases are unfavourable because, if they last long enough, they open the possibility for spontaneous ELMs as seen in our previous experiment [14].

Finally it is interesting to note the behaviour of locked mode detector signals in figure $2 \mathrm{~d}$. During pellet deposition there is a fast swing which is correlated with the spike on the magnetic pickup detector discussed above. After this fast event the locked mode signals slowly relaxes to the pre-pellet value. However this relaxation is not complete and a slow drift is evident during the shown time window indicating that the $3 \mathrm{D}$ equilibrium is evolving on a longer time scale (see the relative amplitude of two locked mode signals).

\section{Pellet fuelling throughput}

The pellet particle throughput is an important parameter of a burning plasma. It provides a link between particle transport at the plasma periphery (where pellets are deposited) on the one side, and burnup fraction of the fusion reactor on the other side. In our case of quasi-stationary plasma the pellet rate is $f_{p e l}=23 \mathrm{~Hz}$ and the fuelling rate is $\Phi_{p e l}=N_{p e l} f_{p e l}=$ $2.8 \times 10^{21}$ atoms/s. This value can be compared with the power normalised to pedestal temperature which gives;

$$
\Phi_{\text {pel }}=0.040 P_{\text {aux }} / T_{e, p e d}
$$

Here $T_{e, p e d}=528 \mathrm{eV}$ is the time averaged electron temperature at the pedestal is as seen in figure $2 \mathrm{~b}$ and $P_{a u x}=$
$5.9 M W$ is the auxiliary heating power. The front coefficient in equation (1) $\alpha=0.040$ represents a ratio of particle to heat flux and its value depends on detailed transport physics of particle losses. In our previous pellet fuelling experiments with RMPs $\alpha=0.05-0.07[13,14]$, but in these plasmas only ELM mitigation was observed and the particle loss was dominated by ELMs. This ELM mechanism is missing in our present case with full ELM suppression and it is replaced by a new continuous post pellet particle loss. The physics model of this transport process is poorly developed and such work is outside the scope of this letter. Here we just note that the future model of post pellet particle transport should explain how the pellet material is removed from the pellet deposition zone including the part with a positive density gradient $\rho_{\text {pol }}=$ $0.8-0.9$ (figure $2 \mathrm{~g}$ ). This observation likely points to a convective particle transport. Alternative mechanism is the inout transport asymmetry due to stabilisation of micro turbulence in the region of positive density gradient [24] but here the details of inward propagation of the front with zero density gradient have to be elaborated. Independently on the mechanism, the time averaged radial velocity that correspond to this process is $\left\langle v_{r}\right\rangle \sim \Phi_{\text {pel }} /\left(S n_{e, p e d}\right) \sim 2 \mathrm{~m} / \mathrm{s}$, where $\mathrm{S}$ is the plasma surface. This value is in the same ballpark as in pellet fuelling experiments with RMP on MAST [15].

\section{Conclusions}

This letter reports on compatibility of pellet fuelling and ELM suppression by RMP, namely:

- For the first time fuelling pellets are shown to preserve ELM suppression by RMPs at low collisionality

- Individual pellets trigger benign MHD events.

- The existence of ELM suppression with pellets is limited to below a certain pellet rate.

Future work should improve on stationarity of the phase with ELM suppression and pellet fuelling. This might be achieved by using both actuators, pellets and RMPs, in feedback mode.

\section{Acknowledgements}

This work has been carried out within the framework of the EUROfusion Consortium and has received funding from the Euratom research and training programme 2014-2018 and 2019-2020 under grant agreement No 633053 and from the RCUK Energy Programme [grant number EP/P012450/1]. To obtain further information on the data and models underlying this paper please contact PublicationsManager@ukaea.ac.uk. The views and opinions expressed herein do not necessarily reflect those of the European Commission. 


\section{References}

[1] Polevoi A S et al 2017 Nucl. Fusion 57022014

[2] Maruyama S et al 2012 Proc.24th Int. Conf. on Fusion Energy (San Diego, 2012) ITR/P5-24 http://www-

naweb.iaea.org/napc/physics/FEC/FEC2012/index.htm

[3] Evans T E et al 2008 Nucl. Fusion 48024002

[4] Kirk A et al 2012 Phys. Rev. Lett. 108255003

[5] Suttrop W et al 2011 Phys. Rev. Lett. 106225004

[6] Liang Y et al 2010 Plasma Fusion Res. 5 S2018

[7] Loarte A et al 2014 Nucl. Fusion 54033007

[8] Baylor L R et al 2008 Proc. 35th EPS Conf. on Plasma Physics (Hersonissos, Greece 2008) vol 32D (ECA) P 4.098

[9] Evans T E 2008 Implications of topological complexity and Hamiltonian chaos in the edge magnetic field of toroidal fusion plasmas. Chaos, Complexity And Transport: Theory and Applications. Edited by Chavanis Piere-Henri -. Published by World Scientific Publishing Co. Pte. Ltd., pp. 147-176

[10] Evans T E et al 2013 Journal of Nucl. Materials 438 S11

[11] Lang P T et al 2012 Nucl. Fusion 52023017

[12] Suttrop W et al 2011 Plasma Phys. Control. Fusion 53124014

[13] Valovič M et al 2016 Nucl. Fusion 56066009

[14] Valovič M et al 2018 Plasma Phys. Control. Fusion 60085013

[15] Valovič M et al 2013 Plasma Phys. Control. Fusion 55025009

[16] Valovič M et al 2015 Nucl. Fusion 55013011

[17] Suttrop W et al 2017 Plasma Phys. Control. Fusion 59014049

[18] Evans T E et al 2004 Phys. Rev. Lett. 92235003

[19] Suttrop W et al 2018 Nucl. Fusion 58096031

[20] Lang P T et al 2003 Rev. Sci. Instr. 743974

[21] Garzotti L et al 2019 Nucl. Fusion 59026006

[22] Askinazi L.G. et al 1993 Phys. Fluids B 52420

[23] Gohil P et al 2001 Phys. Rev. Lett 86644

[24] Valovič M et al 2012 Nucl. Fusion 52114022

[25] Garzotti L et al 2014 Plasma Phys. Control. Fusion 56035004 